\title{
Pretreatment of Rapeseed Meal Increases Its Recalcitrant Fibre Fermentation and Alters the Microbial Community in an in Vitro Model of Swine Large Intestine
}

\section{Cheng LONG}

Maastricht University https://orcid.org/0000-0002-6603-6685

Koen Venema ( $\nabla$ k.venema@maastrichtuniversity.nl)

https://orcid.org/0000-0001-7046-5127

\section{Research}

Keywords: cell wall polysaccharides, rapeseed meal, pigs, gut microbiota, pectinase, cellulase, swine large intestine model

Posted Date: June 15th, 2020

DOl: https://doi.org/10.21203/rs.3.rs-34955/v1

License: (c) (1) This work is licensed under a Creative Commons Attribution 4.0 International License. Read Full License 


\section{Abstract}

Background: The aim of current study was to investigate whether degradation of rapeseed meal (RSM) which was modified by a cellulase, two pectinase, or alkaline treatment was improved by the swine gut microbiota compared to untreated RSM, and whether the microbiota composition was changed.

Methods: An in vitro study was performed to assess how enzymatic and chemical pretreated rapeseed meal (RSM) influences the fibre fermentation and microbial community in the swine large intestine. RSM was processed enzymatically by a cellulase (CELL), two pectinases (PECT), or chemically by an alkaline (ALK) treatment. 16S rRNA gene sequencing data was performedto evaluate changes in the gut microbiota composition, whereas short chain fatty acid production (ion-chromatography) and non-starch polysaccharides (NSP) composition(using monoclonal antibodies; mAbs) were used to assess fibre degradation.

Results: The results showed that ALK, CELL, PECT1, and PECT2changed microbial community composition, increased the abundance of microbial fibre-degrading enzymes and pathways,andincreased acetic acid, propionic acid, butyric acid, and total SCFA production. The increased genera also positively correlated with SCFA production. The cell wall polysaccharide structuresof RSM shiftedafter ALK, CELL, PECT1, and PECT2 treatment. The degradation of NSPduring the fermentation period was dynamic, and not continuous based on the epitope recognition by mAbs.

Conclusion: This study provides the first detailed analysis of changes in the swine intestinal microbiota due to RSM modified by ALK, CELL, PECT1 and PECT2. ALK, CELL, PECT1 and PECT2 altered microbial community structure, shifted the predicted functional metagenomic profile and subsequently increased total SCFA production. Our findings that ALK, CELL, PECT1 and PECT2increased fiber degradability in RSM could help guide feed additive strategies to improve efficiency and productivity in swine industry. The current study gave insight into how feed enzyme modulate microbial status, which provides good opportunity to develop novel carbohydrase, particularly in swine feed.

\section{Introduction}

Rapeseed meal (RSM), a by-product of rapeseed oil production, is not only a suitable protein source for swine feed but also a potentially energy source. RSM contains 20 to $40 \%$ non-starch polysaccharides (NSP) (Simbaya et al. 1995; Slominski and Campbell 1990). The primary cell walls of RSM consist of pectin and xyloglucan and its cellulose microfibrils are interlinked with xyloglucan via hydrogen bonds forming a stiff network (Carpita and Gibeaut 1993). Pectins are linked to each other and cross-linked between pectin and hemicellulose, and between pectin and cellulose (Broxterman and Schols 2018). Pectins, consisting of homogalacturonan, rhamnogalacturonan, xylogalacturonan, arabinogalactan and arabinan, are the major polysaccharides present in the dehulled rapeseed meal (Eriksson, Andersson, and Åman 1997). In the secondary cell wall of RSM, the main carbohydrates are 4-0-methyglucuronoxylan, xyloglucan, and cellulose. A drawback of using RSM in animal feed is that the complex cell wall 
polysaccharides cannot be utilized by endogenous enzymes from monogastric animals (e.g. pigs), and also can only partly be fermented by the microbial community in the gastrointestinal tract (GIT) of the pig.

Therefore, the animal feed sector seeks opportunities to enhance degradability of NSP of feedstuffs, in order to improve its potential as a nutrient source for domestic animals. Previous research showed that physical processing technologies, such as hammer milling, pelleting, wet-milling, extrusion, and mild hydrothermal acid treatment, had limited effect on recalcitrant NSP structures (de Vries et al. 2013; De Vries et al. 2012). As a result, more efficient solutions are needed to modify the cell wall architecture and allow the gut microbiota to utilize the complex carbohydrates. Former research has shown that NSPdegrading enzymes, such as cellulase and pectinases, could open the cell wall structure and improve NSP degradability (Giraldo et al. 2008; Giraldo et al. 2007). Previous studies showed that addition of pectolytic enzymes improved degradability of NSP of RSM in vitro (Pustjens et al. 2012) and in broilers (Pustjens et al. 2014; De Vries et al. 2014). However, none of the above studies investigated how the gut microbiota was affected by the modified RSM or by the feed enzymes. It is important to know this, as NSP can only be fermented by microbes. Bindelle et al. (Bindelle et al. 2011) demonstrated that NSP-degrading enzymes increased abundances of cellulolytic Ruminococcus- and xylanolytic Clostridium-like bacteria and altered fermentation patterns of barley cultivars and wheat products. Torok and colleagues (Torok et al. 2008) investigated changes in gut microbial population in response to the supplementation of an NSP-degrading enzyme (containing $\beta$-glucanase, xylanase, and protease activities) in a barley-based diet in chickens, and the results showed that microbial composition revealed distinct clusters correlating with un-supplemented and enzyme supplemented birds. Previous research reported that the pretreatment of feed stuffs with carbohydrases can cause the release of reducing sugars and other hydrolysis materials, promoting chemotactic response in specific bacteria, and stimulating their attachment to feed particles, and thereby growth of these microbes (Giraldo et al. 2007; Beauchemin et al. 2003; Ribeiro et al. 2015; Ribeiro et al. 2018).

In the present study, RSM (predigested with digestive enzymes) was treated independently with two kinds of pectinases (PECT1 and PECT2), one cellulase (CELL), or alkaline (ALK), and afterwards the untreated and treated RSM preparations were fermented in the Swine Large Intestine in vitro Model (SLIM) (Long, de Vries, and Venema 2020). The aim of the current study was to investigate whether fermentation by the swine gut microbiota of treated RSM was improved compared to untreated RSM, and whether the microbiota composition and activity were changed.

\section{Materials And Methods}

\section{Substrate preparation}

Rapeseed meal (Brassica napus, Cargill N.V., Antwerp, Belgium; 2011) was obtained from a commercial feed mill (Agrifirm B.V., Utrecht, the Netherlands). Preparation method I (predigestion of RSM after carbohydrase or alkaline treatment) [Figure 1]: to $200 \mathrm{~g}$ of RSM $40 \mathrm{~mL}$ 10*gastric electrolyte concentrate 
solution (GES, $310 \mathrm{~g}$ sodium chloride, $110 \mathrm{~g}$ potassium chloride, $15 \mathrm{~g}$ calcium chloride di-hydrate, and $4840 \mathrm{~g}$ ultrapure water) and $360 \mathrm{~mL}$ ultrapure water were added. The $\mathrm{pH}$ was adjusted to 5.5 and then nothing (CON), $10 \mathrm{~mL}$ of alkaline (ALK, $6 \mathrm{M} \mathrm{NaOH})$, or the following carbohydrases were added CELL (Accellerase 1000, Sigma-Aldrich, Missouri, United States), PECT1 (Pectinex Ultra SP, Novozymes A/S, Bagsvaerd, Denmark), or PECT2 (Multifect Pectinase, DuPont Industrial Biosciences, Genencor division, Rochester, NY). Enzyme preparations were incubated at $37^{\circ} \mathrm{C}$ for $2 \mathrm{~h}$, with occasional shaking (every $30 \mathrm{~min}$ ), while ALK was incubated overnight at $4{ }^{\circ} \mathrm{C}$. Enzyme preparations were then heated at $100{ }^{\circ} \mathrm{C}$ for 5 min to inactive enzymes. Afterwards, for all five samples, $120 \mathrm{~mL}$ GES was added and pH adjusted to 3 to continue with the gastric incubation according to the predigestion protocol as described elsewhere (Sáyago-Ayerdi, Zamora-Gasga, and Venema 2017). After predigestion, the slurry was centrifuged $\left(8.000 \mathrm{~g}\right.$, at $4{ }^{\circ} \mathrm{C}$, for 20 minutes) and dialysis was performed for the supernatants. For dialysis, a dialyzer (Sureflux, Nipro Europe Group Companies, Mechelen, Belgium) was used with a peristaltic pump to remove small digestion products and water. After reduction of the total volume to $\sim 450-500 \mathrm{~mL}$, supernatant was mixed with pellet, and freeze-dried. Method II (predigestion of RSM before carbohydrase or alkaline treatment) [Figure 1]: four batches of $200 \mathrm{~g}$ RSM were predigested as described before (Sáyago-Ayerdi, Zamora-Gasga, and Venema 2017) and then dialyzed. Afterwards, $55 \mathrm{~mL} 10 * \mathrm{GES}$ was added, and $\mathrm{pH}$ adjusted to 5.5 , after which $10 \mathrm{~mL}$ of CELL, PECT1, PECT2, or ALK treatment commenced, respectively. Enzyme preparations were incubated at $37^{\circ} \mathrm{C}$ for 2 hours with occasional shaking (every $30 \mathrm{~min}$ ), and ALK was incubated overnight at $4{ }^{\circ} \mathrm{C}$. Afterwards, enzyme preparations were heated at 100 ${ }^{\circ} \mathrm{C}$ for 5 min to inactive enzymes, and $\mathrm{pH}$ was neutralized to 6.5-7 with $\mathrm{HCl}$ or $\mathrm{NaOH}$, and the samples were freeze-dried. Samples are differentiated by the suffix _B (for before) or _A (for after) (e.g. PECT1_A) for carbohydrase- or ALK-treatment prior to and after digestion, respectively.

\section{Fermentation in The Swine in vitro Large Intestinal Model (SLIM)}

The setup of SLIM was as follows: a fully computer-controlled in vitro model based on TIM-2 (Minekus et al. 1999) was used to mimic the swine large intestine (Long, de Vries, and Venema 2020). The pH (5.9) was controlled by continuous addition of $2 \mathrm{M}$ sodium hydroxide. Standard ileal efflux medium of pigs (SIEMP) was used to simulate the materials entering the colon. The SIEMP, adapted from (Gibson, Cummings, and Macfarlane 1988) and described in Long et al.(2020) (Long, de Vries, and Venema 2020) contained the following components $(\mathrm{g} / \mathrm{L})$ : 74.6 maize starch, 9.0 xylan, 19.0 pectin, 9.0 amylopectin, 9.0 arabinogalactan, 9.0 arabinoxylan, 9.0 xyloglucan, 31.5 Tween $80,43.7$ casein, 0.7 ox-bile, 43.7 bactopepton, $4.7 \mathrm{~K}_{2} \mathrm{HPO}_{4} .3 \mathrm{H}_{2} \mathrm{O}, 0.009 \mathrm{FeSO}_{4} .7 \mathrm{H}_{2} \mathrm{O}, 8.4 \mathrm{NaCl}, 0.8 \mathrm{CaCl}_{2} \cdot 2 \mathrm{H}_{2} \mathrm{O}, 0.7 \mathrm{MgSO}_{4} .7 \mathrm{H}_{2} \mathrm{O}, 0.05$ bile, 0.02 haemin and 0.3 cysteine $\cdot \mathrm{HCl}$, plus $1.5 \mathrm{~mL}$ of a vitamin mixture containing (per litre): $1 \mathrm{mg}$ menadione, $0.5 \mathrm{mg}$ vitamin B12, $2 \mathrm{mg}$ D-biotin, $10 \mathrm{mg}$ pantothenate, $5 \mathrm{mg} \mathrm{p}$-aminobenzoic, $4 \mathrm{mg}$ thiamine and $5 \mathrm{mg}$ nicotinamide acid. The $\mathrm{pH}$ was adjusted to 5.9. Dialysis liquid contained (per litre): $2.5 \mathrm{~g} \mathrm{~K}_{2} \mathrm{HPO}_{4} .3 \mathrm{H}_{2} \mathrm{O}, 0.005 \mathrm{~g} \mathrm{FeSO}_{4} .7 \mathrm{H}_{2} \mathrm{O}, 4.5 \mathrm{~g} \mathrm{NaCl}, 0.45 \mathrm{~g} \mathrm{CaCl}_{2} .2 \mathrm{H}_{2} \mathrm{O}, 0.05 \mathrm{~g}$ bile, $0.5 \mathrm{~g} \mathrm{MgSO}_{4} .7 \mathrm{H}_{2} \mathrm{O}$ and $0.4 \mathrm{~g}$ cysteine $\cdot \mathrm{HCl}$, plus $1 \mathrm{~mL}$ of the vitamin mixture. All medium components were purchased at Tritium Microbiology (Eindhoven, The Netherlands). The pig fecal inoculum was a standardized microbiota from growing pigs, freshly collected from the floor (48 pens with 6 pigs/pen, Hypor Libra $x$ 
Hypor Maxter, Hendrix Genetics, Boxmeer, The Netherlands), but only material from the top was selected (so not toughing the floor). Feces was pooled and mixed with dialysate as described before (Long, de Vries, and Venema 2020).

In order to create a complete anaerobic environment, SLIM with $90 \mathrm{~mL}$ dialysate in each of the 4 individual units was flushed with gaseous nitrogen for at least 3 hours before incorporating the standardized microbiota. Thirty $\mathrm{mL}$ of the standardized microbiota was added to each SLIM-unit, making the total volume $120 \mathrm{~mL}$. Figure 1 shows the experimental set-up for fibre addition to SLIM. The microbiota was adapted to the model with SIEMP for 16 hours. During the adaptation phase, SIEMP was added into each SLIM-unit at a rate of $2.5 \mathrm{~mL} / \mathrm{h}$ through the feeding syringe. At the end of the adaptation period, a 2-hour starvation period was performed, which was used to allow all the carbohydrates within SIEMP to be fermented. Afterwards, a shot of 5 grams of the different RSMs was given to the system at time point 0 hours, and incubation was continued for $24 \mathrm{~h}$ after that (Fig. 1).

\section{Sample Collection}

Samples from lumen and spent dialysate were collected at time point $0,0.5,1,2,4,6,8$, and $24 \mathrm{~h}$ (t0, t0.5, $\mathrm{t} 1, \mathrm{t} 2, \mathrm{t} 4, \mathrm{t} 6, \mathrm{t} 8$, and $\mathrm{t} 24)$. They were snap-frozen in liquid nitrogen and stored until analyses. Lumen samples were used to analyze microbiota composition and polysaccharides structures, and both lumen and dialysis samples were analyzed for short chain fatty acid (SCFA) concentrations.

\section{Sequencing of the V3-V4 region of the 16S rRNA gene}

Microbial DNA extraction and sequencing of the V3-V4 region of the 16S rRNA gene were performed by BaseClear B.V. (Leiden, The Netherlands). Briefly, genomic DNA extraction was performed using the QuickDNA $^{\text {TM }}$ Fecal/Soil Microbe Miniprep Kit (Zymo Research, California, USA) according to the manufacturer's instructions. Barcoded amplicons from the V3-V4 region of 16S rRNA genes were generated using a 2step PCR. 10-25 ng genomic DNA was used as template for the first PCR with a total volume of $50 \mu \mathrm{L}$ using the 341F (5'-CCTACGGGNGGCWGCAG-3') and the 785R (5'-GACTACHVGGGTATCTAATCC-3') primers (Klindworth et al. 2013) appended with Illumina adaptor sequences. PCR products were purified (QIAquick PCR Purification Kit, Venlo, The Netherlands) and the size of the PCR products were checked on a Fragment analyzer (Advanced Analytical, Ankeny, US) and quantified by fluorometric analysis. Purified PCR products were used for the 2nd PCR in combination with sample-specific barcoded primers (Nextera XT index kit, Illumina, city, CA, USA). Subsequently, PCR products were purified, checked on a Fragment analyzer and quantified, followed by multiplexing, clustering, and sequencing on an Illumina MiSeq with the paired-end (2x) 300 bp protocol and indexing. The sequencing run was analyzed with the Illumina CASAVA pipeline (v1.8.3) and demultiplexed based on sample-specific barcodes.

\section{Bioinformatics Analysis}


The demultiplexed raw sequences obtained from BaseClear were processed using the QIIME2 pipeline (Bolyen et al. 2019). In short, reads were imported and quality filtered and dereplicated with q2-dada2 (Callahan et al. 2016). Next, dada2 was performed with paired-end reads and truncations parameters were as follows: the first 17 and 14 base pairs were trimmed off in forward and reverse reads, respectively. And at position 280 base pairs the fragment was truncated in forward reads, and at position 230 base pairs for the reverse reads. The processed sequences were used for all the downstream analyses. Alpha-diversity (Shannon index) and $\beta$-diversity (weighted and unweighted UniFrac) were analyzed by the q2-phylogeny plugin (https://github.com/qiime2/q2-diversity).

Phylogenetic Investigation of Communities by Reconstruction of Unobserved States, PICRUSt2. The PICRUSt2 software (Douglas et al. 2019) was used to predict microbial functional abundances based on marker gene sequences. KEGG database was used to predict the results.

\section{Chemical Analyses}

Short-chain fatty acids analyses. Samples from lumen and dialysate were analyzed by Brightlabs (Venlo, The Netherlands) for determination of concentrations of SCFA. lon exclusion chromatography (IEC) was applied on an 883 Ion Chromatograph (IC; Metrohm, Switzerland), using a Transgenomic IC Sep ICE-ION300 column ( $30 \mathrm{~cm}$ length, $7.8 \mathrm{~mm}$ diameter and $7 \mu \mathrm{m}$ particles) and a MetroSep RP2 Guard. The mobile phase consists of $1.5 \mathrm{mM}$ aqueous sulphuric acid. Samples were centrifuged $(21,000 \mathrm{~g}, 10 \mathrm{~min})$ and the clear supernatant was filtered through a $0.45 \mu \mathrm{m}$ PFTE filter and diluted with mobile phase (for lumen 1:5, for dialysate 1:2). Ten microliters were loaded on the column by an autosampler 730 (Metrohm). Molecules were eluted according to their pKa.A column flow rate of $0.4 \mathrm{ml}^{*} \mathrm{~min}^{-1}$ was used. The temperature of the column was $65^{\circ} \mathrm{C}$. The organic acids were detected using suppressed conductivity detection

\section{Glycome profiling}

Sample preparation. Lumen samples from each time point and treatment were freeze-dried, after which they were dissolved at $1 \mathrm{mg} / \mathrm{mL}$ in deionized water, and stored at $-20^{\circ} \mathrm{C}$ as stock solutions.

Monoclonal antibodies (mAbs). mAbs were obtained as hybridoma cell culture supernatants from CarboSource (Atlanta, GA, USA) [http://www.carbosource.net/].

ELISA. The ELISA protocol was slightly modified from Pattathil et al. (2010) (Pattathil et al. 2010). In brief, samples prepared above were applied ( $50 \mu \mathrm{L}$ of $100 \mu \mathrm{g} / \mathrm{mL}$ in deionized water per well) to Costar 3598 96-well plates (Corning Life Sciences, city, country) and were dried to the well surfaces by evaporation overnight at $37^{\circ} \mathrm{C}$. Control wells contained deionized water. The plates were blocked with $200 \mu$ of $1 \%(w / v)$ bovine serum albumin (BSA) in Tris-buffered saline (50 mM Tris-HCl, pH 7.6, containing $100 \mathrm{mM}$ sodium chloride) for $1 \mathrm{~h}$. Blocking agent was removed by aspiration, and $50 \mu \mathrm{l}$ of undiluted hybridoma supernatant were added to each well and incubated for $1 \mathrm{~h}$ at room temperature. 
Supernatant was removed and wells were washed three times with $200 \mu$ of $0.1 \%(w / v)$ BSA in Trisbuffered saline (wash buffer). Peroxidase-conjugated goat anti-mouse lgG, anti-mouse lgM, goat anti-rat IgG, or goat anti-rat IgM antibodies (Sigma-Aldrich), depending on the primary antibody used, were diluted 1:5,000 in wash buffer, and $50 \mu \mathrm{l}$ were added to each well and incubated for $1 \mathrm{~h}$. Wells were then washed five times with $200 \mu \mathrm{l}$ of wash buffer. Next, 3,3',5,5'-tetramethylbenzidine (TMB) solution (Sigma-Aldrich, St. Louis, USA) was freshly prepared according to the manufacturer's instructions, and $50 \mu \mathrm{L}$ were added to each well. After $20 \mathrm{~min}$, the reaction was stopped by adding $50 \mu \mathrm{l}$ of $0.5 \mathrm{~N}$ sulfuric acid to each well. The OD of each well was read at a wavelength of $450 \mathrm{~nm}$ using a Multi-mode microplate reader (BioTek Synergy HTX, Abcoude, The Netherlands).

Polysaccharide panel screening. Polysaccharide panel screening of mAbs was carried out by ELISA against all lumen samples immobilized to 96 -well plates. Duplicate preparations of each polysaccharide were used for all experiments reported here. Binding data was visualized in R via ComplexHeatmap package (Gu, Eils, and Schlesner 2016).

\section{Statistics}

Kruskal-Wallis Rank Sum Test (one-way ANOVA on ranks) was applied to compare a-diversities (Shannon index) among different RSM treatments and time points, and Wilcoxon Rank Sum Test was used for pairwise comparison in R version 3.5.3 (https://www.r-project.org/). Bonferroni was used to correct $P$ values. Permutational multivariate analysis of variance (PERMANOVA; REFERENCE) was performed to test the significance of $\beta$-diversity distances in QIIME2 (weighted and unweighted UniFrac) between nonprocessed and processed RSM. The results were visualized in $R$ ( $R$ version 3.5.3).

The amplicon sequence variant (ASV) table (feature table of QIIME2) was normalized and filtered in R, and statistical analysis and visualizations were performed in R and STAMP (Parks, Tyson et al. 2014). The table was normalized via division by the sum of sequences in a given sample and multiplied by the minimum sum across all samples. Relative abundances were filtered as follows: values below a relative abundance threshold of $0.01 \%$ were not taken into account; taxa with a median relative abundance $<1 \%$ in all groups were not considered for statistical analysis. White's non-parametric t-test was applied to compare between $\mathrm{CON}$ and treatments. $P$-values were corrected using the Benjamini-Hochberg method. A $q$-value (corrected $P$-value) $<0.05$ was considered significant.

Pearson correlations between continuous meta-variables and taxonomic variables were calculated and visualized in R. Parameters were set as follows: Missing values for meta-variables were handled as NO imputation (replacing missing data with substituted); zeros were kept for the calculation of correlation; a minimum number of $0.1 \%$ was considered for calculation; a minimum of 4 paired observations were required for calculation of correlations. $P$-values were corrected using the Benjamini-Hochberg method. A q-value (corrected P-value) $<0.05$ was considered significant.

SCFA production between CON and the treated RSM substrates were compared and visualized in R. 


\section{Results}

\section{Characteristics of non-processed and processed RSM}

A comprehensive set of 155 plant cell wall glycan-directed monoclonal antibodies was used to screen untreated RSM by a ELISA-based assay (Pattathil et al. 2010; Pattathil et al. 2012), and 34 antibodies reacted with RSM (data not shown). These were subsequently used in the current study to obtain information on the presence and relative abundance of specific epitopes that are characteristic of the different types of polymers in untreated RSM and RSM processed by ALK, CELL, PECT1, and PECT2.

Figure 2 shows that both increases and decreases in epitope recognition occurred in ALK, CELL, PECT1, and PECT2 compared to CON. Samples from after (_A) and before (_B) predigestion clustered together according to each treatment, which indicated_A and _B from the same treatment had similar epitope accessibility. ALK strongly increased binding of non-fucosylated XG mAbs, while CELL, PECT1, and PECT2 led to disappearance of those compared to CON, regardless of _A and _B treatment. All the treatments increased the binding of "Linseed Mucilage RG-I group" directed mAbs, but had little effect on Xylan-2 and RG-Ic group compared to CON. Binding of MAC204 (AG-1), which is binding to gum tragacanth and to lettuce and green tomato RG-I preparations (arabinogalactan), disappeared with ALK_A, ALK_B, and CELL_B, while increased binding of CCRC-M107 (AG-2), which binds to linear and branched arabinans and RG-I preparations from diverse plants but does not bind to larch arabinogalactan (Pattathil et al. 2010), was observed in ALK_A and ALK_B. CELL_A, CELL_B, PECT1_A, PECT1_B, and PECT2_B led to disappearance of the binding of mAbs of "pectic backbone group" and CCRC-M 133, which also binds to linear and branched arabinans and RG-I preparations from diverse plants but do not bind to larch arabinogalactan. ALK, PECT1, and PECT2 increased binding of mAbs directed against the arabinogalactan side chains of RG-I (RG-I/AG).

\section{ALK, CELL, PECT1, and PECT2 significantly changed microbiota composition compared to CON}

To determine the changes in composition of the gut microbiota fed with a shot of $5 \mathrm{~g}$ CON, ALK, CELL, PECT1, or PECT2, a comparison of microbiota based on sequencing the V3-V4 region of the 16S rRNA gene was performed. Shannon indeces significantly decreased at $t 4, t 6, t 8$ and $t 24$, compared to that of t0 (Figure S1A). When data from all of the time points were pooled, there were no significant differences among CON, ALK, CELL, PECT1, and PECT2 in Shannon index (Figure S1B). Phylogeny based UniFrac methodology was then used to compare the $\beta$-diversity of the microbial communities between microbiota fed with non-processed and processed RSM. Unweighted UniFrac analysis (Fig. 3) shows that samples from processed RSM (ALK, CELL, PECT1, and PECT2) significantly $(P=0.004)$ separated from nonprocessed RSM (CON), and samples from different processed method clustered together. Samples from CON, ALK, CELL, PECT1, and PECT2 all clustered together $(P=0.125)$ with respect to weighted UniFrac (Figure S2).

There were no significant differences between microbiota fed with RSM predigested before or after carbohydrase or ALK treatment with respect to both a-diversity (Figure S3), or $\beta$-diversity (data not 
shown), which indicated predigesting before or after processing RSM had little effect on microbiota composition. Glycome profiling of RSM (Fig. 2) also shows that samples from after and before processing clustered together according to each treatment, which indicated their polysaccharide compositions were similar to each other. Therefore, they were treated as duplicates in the following (microbial relative abundance) analyses.

Relative abundances of taxa within the pig microbiotas fed with non-processed and processed RSM were compared to identify significantly different bacterial taxa. At genus level (Fig. 4), seven genera were significant higher in relative abundance after ALK, CELL, PECT1, and PECT2 treatment compared to CON. These were Ruminococcaceae NK4A214 group, Ruminococcaceae UCG002, Ruminococcaceae UCG-005, Roseburia, Anaerotruncus, Bifidobacterium, Christensenellaceae R-7 group, and Selenomonas. For genera Christensenellaceae $R-7$ group and Ruminococcaceae UCG-005, their relative abundances were also higher in ALK and PECT1 compared to CELL. Instead, the relative abundances of Prevotella 7, an unclassified genus from Prevotellaceae, and Prevotellaceae UCG-001 were significantly decreased after feeding ALK, CELL, PECT1, and PECT2 compared to microbiota fed with CON. The relative abundance of Succinivibrionaceae UCG-001 was significant higher in ALK and PECT1 compared to CON.

\section{PICRUSt2 analyses revealed that microbial functional abundances related to carbohydrate metabolism and SCFA production were significantly increased with processed RSM compared to CON}

PICRUSt2 was performed to the 16S rRNA gene data to predict metagenomic functional profiles. In this study we focused on carbohydrate metabolism related microbial functions (Fig. 5). The relative abundances of fibre degradation pathways, beta-glucosidase [EC:3.2.1.21], beta-mannosidase [EC:3.2.1.25], cellobiose phosphorylase [EC:2.4.1.20], and sucrose phosphorylase [EC:2.4.1.7], were significant higher in ALK, CELL, PECT1, and PECT2 compared to CON, whereas conversely that of alphaL-fucosidase [EC:3.2.1.51] was significant higher in CON compared to ALK, CELL, PECT1, and PECT2. For cellobiose phosphorylase [EC:2.4.1.20] and sucrose phosphorylase [EC:2.4.1.7], the relative abundances in ALK were also significant higher than those of CELL, PECT1, and PECT2.

Six microbial pathways related to fermentation were significant higher in relative abundance when microbiotas were fed with ALK, CELL, PECT1, and PECT2 compared to microbiota fed with CON. These pathways were pyruvate dehydrogenase $\mathrm{E} 1$ component, short-chain fatty acids transporter, mannose-1phosphate guanylytransferase, superpathway of glucose and xylose degradation, sucrose degradation IV, and L-lysine fermentation to acetate and butanoate. The relative abundance of lactose/L-arabinose transport system permease protein was significant higher in ALK, PECT1 and PECT2 compared to CON.

Figure 6 shows that the cumulative acetic, propionic, and butyric acid and total SCFA production were higher in ALK, CELL, PECT1, and PECT2 compared to CON. For acetic acid, more than 2 times greater production was observed when the microbiota was fed with ALK, CELL, PECT1, and PECT2 compared to when the microbiota was fed with CON. The production of propionic, butyric acid and total SCFA in ALK, CELL, PECT1, and PECT2 were more than 1.6 times higher than that in CON, except for propionic (1.3 times), and butyric acid (1.4 times) production in ALK. 
Glycome profiling shows that binding of mAbs in lumen digests were dynamic during the in vitro fermentation in SLIM

To investigate the dynamic changes of polysaccharides structure in CON, ALK, CELL, PECT1, and PECT2 during in vitro fermentation, a time series of sampling was performed and the set of $34 \mathrm{mAbs}$ was used to screen the lumen digests. Figure 7 shows that no or few binding signals were observed in Nonfucosylated XG, AG-2, and Pectic Backbone mAbs upon feeding CON, ALK, CELL, PECT1, and PECT2 at all time points.

No binding of CCRC-M83 that specifically bind to Linseed Mucilage RG-I was observed at t0 in all treatments (just prior to addition of the fibre shots), and binding signals appeared afterwards. For ALK, binding of CCRC-M83 decreased from t0.5 to t2, increased again at t4, and decreased to the lowest value at t24. For CELL, binding of CCRC-M83 decreased from t0.5 to t2, stabilized from t 4 to t8, and decreased to the lowest value at t24. For CON, binding increased from to. 5 to $t 1$, decreased from t 2 to $t 6$, increased at t8, and decreased to the lowest value at t24. For PECT1, binding increased from t 0.5 to $t 4$, and then decreased progressively until t24. For PECT2, binding increased slightly from t0.5 to t8, and then decreased to the lowest value at $\mathrm{t} 24$.

In terms of Xylan-2 recognizing mAb (i.e. CCRC-M105), weak bindings were detected at all time points after t0 with CELL and CON. Increased binding of CCRC-M105 was observed from t0.5 to t 1 in ALK and the binding strength decreased from $t 2$ to $t 4$, slightly increased again at t6, and then decreased until t24. For PECT1, increased binding of CCRC-M105 was observed from t0.5 to t6, which decreased afterward until t24. Binding for PECT2 was dynamic from t0.5 to t24, but the lowest binding was observed at $\mathrm{t} 24$.

As for the RG-Ic recognizing mAb (i.e. CCRC-M30), binding of CCRC-M30 was lower in ALK, PECT1, and PECT2 according to each time point compared to CELL and CON, but binding over time was dynamic. With respect to the AG-4 mAbs (recognizing arabinogalactans), weak and dynamic binding was observed at each time point in all treatments.

With respect to RG-1/AG mAbs, more active mAbs were observed compared to other groups of mAbs in all treatments. Within RG-1/AG mAbs, binding strengths of CCRC-M25 and CCRC-M60 were stronger than other RG-I/AG mAbs in all treatments, and their binding strengths were fluctuating during the whole fermentation period and still existed at $\mathrm{t} 24$ in all treatments.

\section{Correlation between microbiota abundance and SCFA production and mAb binding}

Pearson correlation analyses were performed to investigate the relationship between the relative abundance of microbial genera and SCFA production at each time point (Fig. 8). Seven genera (Bifidobacterium, Collinsella, Denitrobacterium, Olsenella, Coriobacteriaceae.1, Bacteroidales S24-7 group.2, and Acetitomaculum) had significant negative correlation with propionic acid, butyric acid, valeric acid and total SCFA production. Within these, Bacteroidales S24-7 group.2, Olsenella, Coriobacteriaceae.1, and Acetitomaculum also significantly negatively correlated with acetic acid. Eight 
genera (Bacteroidales S24-7 group.1, Prevotella 9, Faecalibacterium, Ruminococcaceae UCG-005, Ruminococcus 2, Selenomonas, Succinivibrio, and Succinivibrionaceae UCG001) significantly positively correlated with acetic acid, propionic acid, butyric acid, valeric acid and total SCFA production. Within these, Ruminococcus 2 and Succinivibrio also had significant positive correlation with caproic acid. Bacteroidales S24-7 group, Sarcina, and Oribacterium had significant positive correlation with propionic acid, butyric acid, valeric acid and total SCFA production. Roseburia, Ruminococcaceae NK4A214 group and Ruminococcaceae UCG-002 significantly positively correlated with acetic acid, propionic acid, butyric acid and total SCFA production. Prevotella 7 significantly positively correlated with propionic acid, valeric acid, caproic acid, and total SCFA production.

The correlation between binding of mAbs and relative abundance of microbial genera was also analyzed. Within the mAbs that recognizing non-fucocylated XG, CCRC-M93 had significant negative correlation with Bacteroidales S24-7 group.1, Christensenellaceae R-7 group, [Eubacterium] nodatum group, Blautia, Lachnospira, Lachnospiraceae NK3A20 group, Roseburia, Ruminococcaceae UCG-005, Ruminococcus 2, Subdoligranulum, Catenibacterium, Catenisphaera, Succiniclasticum, and Succinivibrionaceae UCG-001. CCRC-M96 significantly negatively correlated with Bacteroidales S24-7 group.2 and Acetitomaculum, while it significantly positively correlated with Anaerotruncus. CCRC-M99 had significant negative correlation with Dorea and Ruminococcus 2, whereas it had significant positive correlation with Megasphaera. CCRC-M104 had significant positive correlation with Prevotellaceae UCG-001, while it negatively correlated with Ruminococcaceae UCG-002.

$\mathrm{MH} 4.2 \mathrm{~A} 4$ that recognizes linseed mucilage RG-I had significant negative correlations with Bacteroidales S24-7group.1, Ruminococcaceae NK4A214 group, and Ruminococcaceae UCG-005.

CCRC-M105 that binds to Xylan-2 had significant negative correlations with Prevotellaceae.1, Family XIII UCG-001, Acidaminococcus, and Megasphaera, whereas it positively correlated with Ruminococcus 2 and Subdoligranulum.

CCRC-M30, binding to RG-Ic, had significant positive correlations with Denitrobacterium, Olsenella, Bacteroidales S24-7 group.2, and Acetitomaculum, while it significantly negatively correlated with Roseburia, Faecalibacterium, Ruminococcaceae UCG-002, Ruminococcaceae UCG-005, Selenomonas, and Succinivibrionaceae UCG-001.

MAC204, that recognizing AG-1, significantly negatively correlated with Denitrobacterium, Olsenella, Bacteroidales S24-7 group.2, Acetitomaculum, and Roseburia, while it had significant positive correlations with Lactobacillus, Sarcina, Roseburia, Ruminococcaceae NK4A214 group, Ruminococcaceae UCG002, Ruminococcaceae UCG-005, and Selenomonas.

CCRC-M133, binding to AG-2, significantly negatively correlated with Denitrobacterium, Prevotella 7, Prevotellaceae UCG-001, Acetitomaculum, and Subdoligranulum, while it positively correlated with Ruminococcaceae UCG-002. 
CCRC-M131, that binds to pectic backbone, had significant negative correlation with Denitrobacterium, Olsenella, Bacteroidales S24-7 group.2, Prevotellaceae UCG-001, Acetitomaculum, and Subdoligranulum, while it positively correlated with Lactobacillus, Anaerotruncus, and Ruminococcaceae UCG-002.

With respect to mAbs binding to AG-4, CCRC-M78 had significant negative correlation with Bifidobacterium, and Christensenellaceae $R$-7 group, whereas it significantly positively correlated with Prevotellaceae UCG-001. CCRC-M91 had significant negative correlation with Denitrobacterium, Olsenella, and Acetitomaculum, and CCRC-M91 and CCRC-M92 significantly positively correlated with Lactobacillus. JIM13 had significant negative correlation with Subdoligranulum.

As for mAbs binding to RG-I/AG, they had significant negative correlation with Bacteroidales $524-7$ group (CCRC-M32), Bacteroidales S24-7 group.2 (CCRC-M32 and M125), Lactobacillus (CCRC-M24), [Eubacterium] nodatum group (CCRC-M60), Acetitomaculum (CCRC-M42), Blautia (CCRC-M60), Coprococcus 3 (CCRC-M24), Ruminococcaceae NK4A214 group (CCRC-M32, M41, M44, and M60), Ruminococcaceae UCG-002 (CCRC-M44), Ruminococcaceae UCG-005 (CCRC-M32, M44, and M60) Subdoligranulum (CCRC-M 42), and Succiniclasticum (CCRC-M125). Significantly positively correlations were observed with Olsenella (CCRC-M44), Bacteroidales S24-7 group (CCRC-M128), Prevotella 7 (CCRCM24 and M128), Prevotellaceae UCG-001 (CCRC-M 24 and M128), Prevotellaceae (CCRC-M128), Lactobacillus (CCRC-M42), Lachnospira (CCRC-M128), Ruminococcus 2 (CCRC-M128), Subdoligranulum (CCRC-M128), and Succinivibrio (CCRC-M128).

\section{Discussion}

\section{Cell wall polysaccharides composition of differently pretreated RSM}

Cell wall polysaccharides of RSM (Brassica napus) were comprehensively studied by chemical methods (Pustjens et al. 2013), which showed that they generally consisted of arabinan, homogalacturonan, Rhamnogalacturoan I (RG-I), type II arabinogalactan (AG), and xyloglucan (XG) (Siddiqui and Wood 1977). In a previous study (Long, de Vries and Venema submitted), we determined the monosaccharide constituent composition, which was in line with Pustjens et al. (2013). Our current findings with the mAbs are also in line with this, since nonfucosylated XG-, RG-I, pectic backbone-, and AG-recognizing mAbs bound to CON. By similar reasoning, xylan was also detected in CON, which was not reported before, although it is not entirely clear how much cross-reactivity the mAbs show.

ALK treatment intensively increased binding of mAbs that specifically bind to nonfucosylated XG (Fig. 2). This is consistent with previous finding that alkali could extract XG from hemicellulose (Pustjens et al. 2013), which led to $X G$ being detected by the mAbs in the current study. Previous research also demonstrated that $X G$ is linked via hydrogen bridges to the surface of cellulose microfibrils, which is extractable with alkali, but not accessible by enzyme (Pauly et al. 1999). However, enzymatic treatments (PECT1, PECT2, and CELL) led to a disappearance of binding nonfucosylated XG-specific mAbs 
compared to CON, which was unexpected. It could be that XG might become entrapped by other cell wall structures after pectinase or cellulase treatment. It is unknown whether this kind of the hidden phenomenon would reduce the degradability of XG.

Enzymatic treatments increased binding of linseed mucilage RG-I-specific mAbs and some mAbs directed against the arabinogalactan side chains of RG-I (RG-I/AG), and reduced binding of some mAbs directed to pectic backbone, which indicated that PECT1 and PECT2 broke down pectic backbone and exposed RG-I and its arabinogalactan side chains. These results are supported by a previous study showing that PECT1 and PECT2 degraded pectic backbone and released RG-I and its arabinogalactan side chains (Pustjens et al. 2013). Moreover, RG-I attached to cellulose microfibrils can be released by cellulase (Oechslin, Lutz, and Amadò 2003), while at the same time it might block the accessibility of pectic backbone because of the shifting of the polysaccharides structure.

ALK treatment led to the disappearance of binding of mAbs that specifically bind to AG-1 while it increased binding of mAbs that specifically binding to AG-2, which indicated that ALK could cause arabinogalactan to be physically entrapped in other cell wall structures, while linear and branched arabinan become accessible (Pattathil et al. 2010).

They were lower in binding strengths of mAbs in AG-4 from ALK_B, PECT1_B, PECT2_B, and CELL_B, compared to ALK_A, PECT1_A, PECT2_A, and CELL_A, respectively. This indicated that small fragments were produced in AG-4 group after enzymatic and chemical treating RSM, which were dialyzed out in ALK_B, PECT1_B, PECT2_B, and CELL_B during the subsequent predigestion treatment. Overall, both enzymatic and chemical processed RSM can release some polysaccharides but physically entrap or shield some others at the same time.

\section{Community structure of swine microbiota fed with differently pretreated RSM}

The PCoA analysis showed significant difference of both enzymatic and chemical treatments on swine microbial community $(P=0.004)$ compared to $\mathrm{CON}$, based on unweighted UniFrac metric (Fig. 3); and overall there were no significant differences in this metric between treatments (Fig. 3). Dietary fibre is known to have a considerable effect on gut microbiota composition (Yatsunenko et al. 2012; Cotillard et al. 2013; Flint 2012). Glycome profiling of non-processed and processed RSM showed that polysaccharides structures were differentially shifted due to ALK, PECT1, PECT2, and CELL treatment in the current study, which were consistent with previous studies (Pustjens et al. 2012; De Vries et al. 2014). These observations might explain the specific genus changes in microbiota composition after microbiotas fed with ALK, PECT1, PECT2, and CELL compare to CON (Fig. 4), despite the differential effect of the different treatments as assessed by the glycome profiling.

In order to understand which microbes were significantly influenced by dietary supplementation of processed RSM, microbial genera were compared among the five treatments (Fig. 4). The relative abundances of Ruminococcaceae NK4A214 group, Ruminococcaceae UCG-002, Ruminococcaceae UCG005, Roseburia, Bifidobacterium, and Christensenellaceae R-7 group were significantly increased in ALK, 
PECT1, PECT2, and CELL (Fig. 4). Research has shown that genera of family Ruminococcaceae contain major (hemi)cellulolytic and pectinolytic species (Nyonyo, Shinkai, and Mitsumori 2014; Hartinger et al. 2019; Pettipher and Latham 1979). Thus, microbes from family Ruminococcaceae play an important role in degrading (hemi)cellulose and pectin in their activity against recalcitrant fibre (Flint et al. 2008; Shinkai and Kobayashi 2007). Previous research demonstrated that the higher the relative abundance of Ruminococcaceae NK4A214 group, the higher the fibre degradability (Hartinger, Gresner, and Südekum 2019; Hartinger et al. 2019). This observation was consistent with the current study, which showed that the SCFA production (which is usually used as evaluation of fibre degradability in vitro) was also higher in ALK, CELL, PECT1, and PECT2 compared to CON. Furthermore, our study also showed that the relative abundance of Ruminococcaceae NK4A214 group, as well as Ruminococcaceae UCG-002 and Ruminococcaceae UCG-005, significantly correlated with acetic acid, propionic acid, butyric acid, and (not surprisingly) total SCFA production. Roseburia is a well-known butyrate-producing bacteria (Duncan et al. 2002), and a primary degrader of $\beta$-mannans (La Rosa et al. 2019). The significant increase in abundance of this genus was accordance with the increased butyric acid production in the current study (Fig. 6), and increased predicted abundance of beta-mannosidases (EC.3.2.1.25) in the processed RSM groups (Fig. 5). Bifidobacterium is suggested to be a common cross-feeding bacteria for sugar utilization (Cockburn and Koropatkin 2016). Previous research has been shown that Bifidobacterium bifidum relies on the presence of a primary degrader in order to grow with either resistant starch or xylan both in vitro (Turroni et al. 2015) and in vivo (Turroni et al. 2016). It has also been reported that numerous Bifidobacterium species grew to higher cell densities accompanied by upregulating their respective saccharolytic pathways when grown in co-culture compared to their growth in monoculture (Milani et al. 2015). This finding was consistent with the current study, where the relative abundance of Bifidobacterium was much higher than that of other genera, especially in ALK (data not shown). Alternatively, synergy existed among genera that were targeting the same substrates, possibly by specializing in degrading different motifs within the molecule.

The relative abundance of Christensenellaceae $R$-7 group increased after enzymatic and chemical treatment, which was consistent with a previous study where rumen microbiota was fed with fibolytic enzyme-treated wheat straw (Ribeiro et al. 2020). Christensenellaceae plays an important role in degrading fibre (Mao et al. 2015) and producing acetic and butyric acid (Morotomi, Nagai, and Watanabe 2012). All the observations above were supported by former reports that feed processed by carbohydrase enzymes could stimulate growth of specific microbes (Wang et al. 2001; Giraldo et al. 2007; Giraldo et al. 2008). It can be speculated that the pretreatment of feed stuffs with carbohydrases causes the release of hydrolysis materials (presumably oligosaccharides), which promote the chemotactic response of specific bacteria, and stimulates their attachment to feed particles, thereby leading to growth of these microbes (Giraldo et al. 2007; Beauchemin et al. 2003; Ribeiro et al. 2015; Ribeiro et al. 2018).

\section{Fibre Degradation And Scfa Production}


Enzymatic and chemical treatment on RSM increased the amount of acetic acid, propionic acid, butyric acid, and thereby total SCFA production (Fig. 6). This observation was consistent with the predicted functional profiles related to carbohydrate metabolism, where the relative abundance of fibre breakdown and fermentation enzymes and pathways increased (Fig. 5). This finding was in accordance with previous studies that addition of pectolytic enzymes improved degradability of non-starch polysaccharides (NSP) of RSM in vitro (Pustjens et al. 2012) and in broilers (Pustjens et al. 2014; De Vries et al. 2014). Giraldo et al. also reported that supplementation of exogenous cellulase increased SCFA production (Giraldo et al. 2007). Previous research demonstrated that supplementation of carbohydrase on feed before feeding could increase microbial protein production, ruminal cellulolytic bacterial numbers, and ruminal fibrolytic activity (Wang et al. 2001). Thus, the findings above indicate that the enzymatic and chemical treatment on RSM could sufficiently open cell wall architecture (refer to Fig. 2), to enable effective accessibility of NSP to bacterial degradation enzymes, and subsequently stimulate expression of microbial saccharolytic pathways.

No bindings of mAbs recognizing non-fucolysated XG were observed in lumen samples after the microbiota was fed with ALK or CON (Fig. 7), which was unexpected as binding signals were seen in the substrates themselves prior to addition to SLIM (Fig. 2). The hypothesis could be entertained that XG was immediately utilized by bacteria before our first sampling time point (after 30 minutes), or that the XG structures were unable to be recognized by the mAbs after supplementing them to lumen, due to entrapment by other molecules. Binding signals still existed at 24 for all mAbs that showed signal at the to. 5 time point, which indicated that these structures cannot be degraded any further or more fermentation time was needed. Glycome profiling showed that binding of mAbs recognizing each polysaccharide structure/epitope were dynamic during the $24 \mathrm{~h}$ fermentation period (Fig. 7). It is not unlikely that certain polysaccharide structure, such as (hemi)cellulose and pectin, were exposed to microbes stage by stage, due to ever increasing degradation of the cell wall structures over time. For instance, bacteria should break down side-chain AG before they can utilize RG-I. However, this hypothesis should be validated in future study.

The increased in abundance of Ruminococcaceae NK4A214 group, Ruminococcaceae UCG-002, and Ruminococcaceae UCG-005 in enzymatic and chemical treated RSM groups showed negative correlations with XG, RG-I, and RG-I/AG (arabinogalactan side chains of RG-I), and positively correlated with AG-1, AG-2, and pectic backbone. These observations suggested that ALK, CELL, PECT1, and PECT2 stimulated these genera to utilize XG, RG-I, and arabinogalactan side chains of RG-I, and exposed AG-1, AG-2, and pectic backbone to other bacteria in the current study. The abundance of Roseburia and Succinivibrionaceae UCG-001 in enzymatic and chemical treated RSM groups had negative correlations with XG and AG-1, which suggested that the treatments on RSM stimulated these genera to degrade XG and AG-1. A possible model of action can be explained by the adhesion theory of Rumen Cellulolytic Bacteria (Miron, Ben-Ghedalia, and Morrison 2001), which supposes that the (in our case) enzymatic and chemical treatments on RSM stimulate attachments of microbes to specific polysaccharides structures (Meale et al. 2014), which lead the bacteria to degrade them. However, the mechanism of exogenous enzymes enhanced degradation of plant cell walls is complex (Luis et al. 2018), with many interrelated 
factors, and requires further studies. Moreover, degradation of fibres requires a plethora of microbial enzymes as indicated for instance by the numerous PUL-loci needed by Bacteroides thetaiotaomicron to breakdown pectin (Luis et al. 2018).

\section{Conclusion}

The present study clearly demonstrated that both enzymatic and chemical pretreatment on RSM shifted its cell wall polysaccharide structure, subsequently altering microbial community composition and functional profile compared to untreated RSM, and eventually increased fibre degradability as evaluated by SCFA production. Furthermore, glycome profiling showed that the abundance of cell wall polysaccharides were dynamically changed during fermentation, and did not continuously decrease during the fermentation period as one might expect. Our findings that ALK, CELL, PECT1 and PECT2 increased fiber degradability in RSM could help guide feed additive strategies to improve efficiency and productivity in swine industry. The current study gave insight into how feed enzyme modulate microbial status, which provides good opportunity to develop novel carbohydrase, particularly in swine feed.

\section{Abbreviations}

RSM: rapeseed meal

CON: control, RSM without treatment

CELL: cellulase, Accellerase 1000, RSM treated with CELL

PECT: pectinase,

PECT1: Pectinex Ultra SP, RSM treated with PECT1

PECT2: Multifect Pectinase, RSM treated with PECT2

ALK: alkaline, $6 \mathrm{M} \mathrm{NaOH}$, RSM treated with ALK

_B: for carbohydrase- or ALK-treatment prior to digestion

_A: for carbohydrase- or ALK-treatment after digestion

SCFA: short chain fatty acids

NSP: non-starch polysaccharides

GIT: gastrointestinal tract

SLIM: Large Intestine in intro Model

GES: gastric electrolyte concentrate solution 
TIM-2: TNO (gastro-) Intestinal Model of colon

SIEMP: standard ileal efflux medium of pigs

mAbs: Monoclonal antibodies

BSA: bovine serum albumin

TMB囚tetramethylbenzidine

ASV: amplicon sequence variant

XG; xyloglucan

RG-I: Rhamnogalacturoan I

AG: arabinogalactan

RG-I/AG: arabinogalactan side chains of RG-I

\section{Declarations}

\section{Availability of data and materials}

Raw sequencing data was submitted to the European Nucleotide Archive under the accession number: PRJEB38485.

\section{Acknowledgements}

For CCRC antibodies "Generation of the CCRC-series of monoclonal antibodies used in this work was supported by a grant from the NSF Plant Genome Program (DBI-0421683)".

For JIM and MAC antibodies "Distribution of the JIM and MAC antibodies used in this work was supported in part by NSF grants DBI-0421683 and RCN 009281.

We thank Sanne Verbruggen, and Jessica Verhoeven for sharing their experience in running TIM-2, and Rob van Dinter for technical support.

\section{Funding}

Cheng Long received a PhD scholarship from China Scholarship Council under the Chinese Government Graduate Student Overseas Study Program.

The study was funded by the Centre for Healthy Eating \& Food Innovation (HEFI) of Maastricht University - campus Venlo. This research has been made possible with the support of the Dutch Province of Limburg. 


\section{Contributions}

K.V. designed and planned the experiment. C.L. performed the data analyses and drafted the manuscript; further K.V. revised and contributed to the manuscript. Both authors read and approved the final manuscript.

\section{Ethics approval and consent to participate}

Since this was not an intervention study, feces collection from the floor of the pins of the pigs did not require ethical approval in accordance with local/national guidelines.

\section{Consent for publication}

Not applicable.

\section{Competing interests}

The authors declare that they have no competing interests.

\section{References}

1. Beauchemin KA, Colombatto D, Morgavi DP, Yang WZ. 'Use of exogenous fibrolytic enzymes to improve feed utilization by ruminants'. Journal of animal science. 2003;81:E37-47.

2. Bindelle J, Pieper R, Montoya CA, Andrew G, Van Kessel. Pascal Leterme. 2011. 'Nonstarch polysaccharide-degrading enzymes alter the microbial community and the fermentation patterns of barley cultivars and wheat products in an in vitro model of the porcine gastrointestinal tract', FEMS Microbiol Ecol, 76: 553-63.

3. Bolyen E, Rideout JR, Dillon MR, Nicholas A, Bokulich CC, Abnet, Gabriel A, Al-Ghalith H, Alexander, Eric J. Alm, Manimozhiyan Arumugam, and Francesco Asnicar. 2019. 'Reproducible, interactive, scalable and extensible microbiome data science using QIIME 2', Nature biotechnology. 1.

4. Broxterman SE, Henk AS. 'Interactions between pectin and cellulose in primary plant cell walls'. Carbohydrate polymers. 2018;192:263-72.

5. Callahan BJ, Paul J, McMurdie MJ, Rosen AW, Han, Amy Jo A, Johnson, and Susan P. Holmes. 2016. 'DADA2: High-resolution sample inference from Illumina amplicon data', Nature Methods, 13: 581.

6. Carpita NC, David MG. 'Structural models of primary cell walls in flowering plants: consistency of molecular structure with the physical properties of the walls during growth'. Plant J. 1993;3:1-30.

7. Cockburn DW, Nicole MK. 'Polysaccharide degradation by the intestinal microbiota and its influence on human health and disease'. J Mol Biol. 2016;428:3230-52.

8. Cotillard A, Kennedy SP, Kong LC, Prifti E, Pons N, Chatelier EL. Mathieu Almeida, Benoit Quinquis, Florence Levenez, and Nathalie Galleron. 2013. 'Dietary intervention impact on gut microbial gene richness', Nature, 500: 585-88. 
9. De Vries S, Pustjens AM, Kabel MA, Kwakkel RP, Gerrits WJJ. 'Effects of processing technologies and pectolytic enzymes on degradability of nonstarch polysaccharides from rapeseed meal in broilers'. Poult Sci. 2014;93:589-98.

10. De Vries S, Pustjens AM, Schols HA, Hendriks WH, Gerrits WJJ. 'Improving digestive utilization of fiber-rich feedstuffs in pigs and poultry by processing and enzyme technologies: A review'. Anim Feed Sci Technol. 2012;178:123-38.

11. de Vries, Sonja AM, Pustjens MA, Kabel S, Salazar-Villanea WH, Hendriks, Walter JJG. 'Processing technologies and cell wall degrading enzymes to improve nutritional value of dried distillers grain with solubles for animal feed: an in vitro digestion study'. J Agric Food Chem. 2013;61:8821-28.

12. Douglas GM, Vincent J, Maffei J, Zaneveld, Svetlana N, Yurgel JR, Brown CM, Taylor C, Huttenhower, Morgan GIL. 2019. 'PICRUSt2: An improved and extensible approach for metagenome inference', bioRxiv. 672295.

13. Duncan SH, Georgina L, Hold A, Barcenilla CS, Stewart, Harry JF. 'Roseburia intestinalis sp. nov., a novel saccharolytic, butyrate-producing bacterium from human faeces'. Int J Syst Evol Microbiol. 2002;52:1615-20.

14. Eriksson I, Andersson R, Per, Åman. 'Extraction of pectic substances from dehulled rapeseed'. Carbohyd Res. 1997;301:177-85.

15. Flint HJ. 'The impact of nutrition on the human microbiome'. Nutr Rev. 2012;70:10-3.

16. Flint HJ, Edward A, Bayer, Marco T, Rincon R, Lamed, White BA. 'Polysaccharide utilization by gut bacteria: potential for new insights from genomic analysis'. Nat Rev Microbiol. 2008;6:121-31.

17. Gibson GLENNR, Cummings JOHNH, Macfarlane GEORGET. 'Use of a three-stage continuous culture system to study the effect of mucin on dissimilatory sulfate reduction and methanogenesis by mixed populations of human gut bacteria'. Appl Environ Microbiol. 1988;54:2750-55.

18. Giraldo LA, Tejido ML, Ranilla MJ, Carro MD. 'Effects of exogenous cellulase supplementation on microbial growth and ruminal fermentation of a high-forage diet in Rusitec fermenters'. Journal of animal science. 2007;85:1962-70.

19. Giraldo LA, Tejido ML, Ranilla MJ, Ramos S, Carro MD. 'Influence of direct-fed fibrolytic enzymes on diet digestibility and ruminal activity in sheep fed a grass hay-based diet'. Journal of animal science. 2008;86:1617-23.

20. Gu Z, Eils R. Matthias Schlesner. 2016. 'Complex heatmaps reveal patterns and correlations in multidimensional genomic data', Bioinformatics, 32: 2847-49.

21. Hartinger T, Gresner N, K-H Südekum. 'In vitro ruminal fermentation characteristics of alfalfa silages in response to different pre-ensiling treatments'. Anim Feed Sci Technol. 2019;258:114306.

22. Hartinger T, Edwards JE. Ruth Gómez Expósito, Hauke Smidt, Cajo JF ter Braak, Nina Gresner, and Karl-Heinz Südekum. 2019. 'Differently pre-treated alfalfa silages affect the in vitro ruminal microbiota composition', Frontiers in Microbiology, 10: 2761.

23. Klindworth A, Pruesse E, Schweer T, Peplies J, Quast C, Horn M, Frank Oliver G. 'Evaluation of general 16S ribosomal RNA gene PCR primers for classical and next-generation sequencing-based diversity 
studies'. Nucleic Acids Res. 2013;41:e1-1.

24. La Rosa S, Leanti ML, Leth L, Michalak ME, Hansen, Nicholas A, Pudlo R, Glowacki G, Pereira, Christopher T, Workman, Magnus $\varnothing$, Arntzen, Phillip BP. 'The human gut Firmicute Roseburia intestinalis is a primary degrader of dietary $\beta$-mannans'. Nature communications. 2019;10:1-14.

25. Long, Cheng S, de Vries. and Koen Venema. 2020. 'Polysaccharide source altered ecological network, functional profile, and short-chain fatty acid production in a porcine gut microbiota', Beneficial microbes.

26. Luis AS, Jonathon Briggs X, Zhang B, Farnell D, Ndeh A, Labourel A, Baslé A, Cartmell N, Terrapon. Katherine Stott. 2018. 'Dietary pectic glycans are degraded by coordinated enzyme pathways in human colonic Bacteroides', Nature microbiology, 3: 210-19.

27. Mao S, Zhang M, Liu J, Zhu W. 'Characterising the bacterial microbiota across the gastrointestinal tracts of dairy cattle: membership and potential function'. Sci Rep. 2015;5:16116.

28. Meale SJ, Karen A, Beauchemin AN, Hristov AV, Chaves, and TA McAllister. 'Board-invited review: opportunities and challenges in using exogenous enzymes to improve ruminant production'. Journal of animal science. 2014;92:427-42.

29. Milani C, Lugli GA, Duranti S, Turroni F, Mancabelli L, Ferrario C, Mangifesta M. Arancha Hevia, Alice Viappiani, and Matthias Scholz. 2015. 'Bifidobacteria exhibit social behavior through carbohydrate resource sharing in the gut', Scientific Reports, 5: 15782.

30. Minekus M, Smeets-Peeters M, Bernalier A, Marol-Bonnin S, Havenaar R, Marteau P, Alric M, Fonty G. and J. H. Huis in't Veld. 1999. 'A computer-controlled system to simulate conditions of the large intestine with peristaltic mixing, water absorption and absorption of fermentation products', App/ Microbiol Biotechnol, 53: 108-14.

31. Miron J, Ben-Ghedalia D, Morrison M. 'Invited review: adhesion mechanisms of rumen cellulolytic bacteria'. J Dairy Sci. 2001;84:1294-309.

32. Morotomi M, Nagai F, and Yohei Watanabe. 'Description of Christensenella minuta gen. nov., sp. nov., isolated from human faeces, which forms a distinct branch in the order Clostridiales, and proposal of Christensenellaceae fam. nov'. Int J Syst Evol Microbiol. 2012;62:144-49.

33. Nyonyo T, Shinkai T, and Makoto Mitsumori. 'Improved culturability of cellulolytic rumen bacteria and phylogenetic diversity of culturable cellulolytic and xylanolytic bacteria newly isolated from the bovine rumen'. FEMS Microbiol Ecol. 2014;88:528-37.

34. Oechslin R, Lutz MV, Renato, Amadò. 2003. 'Pectic substances isolated from apple cellulosic residue: structural characterisation of a new type of rhamnogalacturonan I', Carbohydrate polymers, 51: 30110.

35. Pattathil S, Avci U, Baldwin D, Swennes AG, McGill JA, Popper Z, Bootten T, Albert A, Davis RH, Chennareddy C, Dong R, O'Shea B, Rossi R, Leoff C, Freshour G, Narra R, O'Neil M, York WS. and M. G. Hahn. 2010. 'A comprehensive toolkit of plant cell wall glycan-directed monoclonal antibodies', Plant Physiol, 153: 514-25. 
36. Pattathil S, Avci U, Miller JS, Michael GH. 2012. 'Immunological approaches to plant cell wall and biomass characterization: glycome profiling.' in, Biomass Conversion (Springer).

37. Pauly M, Albersheim P, Darvill A, William SY. 'Molecular domains of the cellulose/xyloglucan network in the cell walls of higher plants'. Plant J. 1999;20:629-39.

38. Pettipher GL, Malcolm JL. 'Production of enzymes degrading plant cell walls and fermentation of cellobiose by Ruminococcus flavefaciens in batch and continuous culture'. Microbiology. 1979;110:29-38.

39. Pustjens AM, de Vries S, Gerrits WJ, Kabel MA, Schols HA, Gruppen H. 'Residual carbohydrates from in vitro digested processed rapeseed (Brassica napus) meal'. J Agric Food Chem. 2012;60:8257-63.

40. Pustjens AM, de Vries S, Schols HA, Gruppen H, Gerrits WJ, Kabel MA. 'Understanding carbohydrate structures fermented or resistant to fermentation in broilers fed rapeseed (Brassica napus) meal to evaluate the effect of acid treatment and enzyme addition'. Poult Sci. 2014;93:926-34.

41. Pustjens AM, Schols HA, Kabel MA, Gruppen H. 'Characterisation of cell wall polysaccharides from rapeseed (Brassica napus) meal'. Carbohydr Polym. 2013;98:1650-6.

42. Ribeiro GO, Ajay Badhan J, Huang KA, Beauchemin W, Yang Y, Wang A, Tsang, Tim AMcAllister. 'New recombinant fibrolytic enzymes for improved in vitro ruminal fiber degradability of barley straw'. Journal of animal science. 2018;96:3928-42.

43. Ribeiro GO, Robert J, Gruninger DR, Jones, Karen A, Beauchemin WZ, Yang Y, Wang DW, Abbott A, Tsang, Tim AMcAllister. 'Effect of ammonia fiber expansion-treated wheat straw and a recombinant fibrolytic enzyme on rumen microbiota and fermentation parameters, total tract digestibility, and performance of lambs'. Journal of animal science. 2020;98:skaa116.

44. Ribeiro GO, Gonçalves LC, Pereira LGR, Chaves AV, Wang Y, Beauchemin KA, and TA McAllister. 2015. 'Effect of fibrolytic enzymes added to a Andropogon gayanus grass silage-concentrate diet on rumen fermentation in batch cultures and the artificial rumen (Rusitec)', Animal, 9: 1153-62.

45. Sáyago-Ayerdi, Sonia G, Victor M, Zamora-Gasga, and Koen Venema. 2017. 'Prebiotic effect of predigested mango peel on gut microbiota assessed in a dynamic in vitro model of the human colon (TIM-2)', Food Research International.

46. Shinkai T, and Yasuo Kobayashi. 'Localization of ruminal cellulolytic bacteria on plant fibrous materials as determined by fluorescence in situ hybridization and real-time PCR'. Appl Environ Microbiol. 2007;73:1646-52.

47. Siddiqui IR, Peter JW. 'Carbohydrates of rapeseed: a review'. J Sci Food Agric. 1977;28:530-38.

48. Simbaya J, Slominski BA, Rakow G, Lloyd D, Campbell R, Keith Downey, Milton Bell J. 'Quality characteristics of yellow-seeded Brassica seed meals: Protein, carbohydrate, and dietary fiber components'. J Agric Food Chem. 1995;43:2062-66.

49. Slominski BA, Lloyd DC. 'Non-starch polysaccharides of canola meal: Quantification, digestibility in poultry and potential benefit of dietary enzyme supplementation'. J Sci Food Agric. 1990;53:175-84.

50. Torok VA, Kathy Ophel-Keller M, Loo, Robert JH. 'Application of methods for identifying broiler chicken gut bacterial species linked with increased energy metabolism'. Appl Environ Microbiol. 
2008;74:783-91.

51. Turroni F, Milani C, Duranti S, Mancabelli L, Mangifesta M, Viappiani A, Lugli GA, Ferrario C, Gioiosa L. Alberto Ferrarini. 2016. 'Deciphering bifidobacterial-mediated metabolic interactions and their impact on gut microbiota by a multi-omics approach', ISME J, 10: 1656-68.

52. Turroni F, Özcan E, Milani C, Mancabelli L, Viappiani A, van Sinderen D, Sela D. Marco Ventura. 2015. 'Glycan cross-feeding activities between bifidobacteria under in vitro conditions', Front Microbiol, 6 : 1030.

53. Wang Y, McAllister TA, Rode LM, Beauchemin KA, Morgavi DP, Nsereko VL, Iwaasa AD, Yang W. 'Effects of an exogenous enzyme preparation on microbial protein synthesis, enzyme activity and attachment to feed in the Rumen Simulation Technique (Rusitec)'. Br J Nutr. 2001;85:325-32.

54. Yatsunenko T, Rey FE, Manary MJ, Trehan I, Dominguez-Bello MG, Contreras M, Magris M, Hidalgo G, Baldassano RN, Andrey PA. 'Human gut microbiome viewed across age and geography'. Nature. 2012;486:222-27.

\section{Figures}

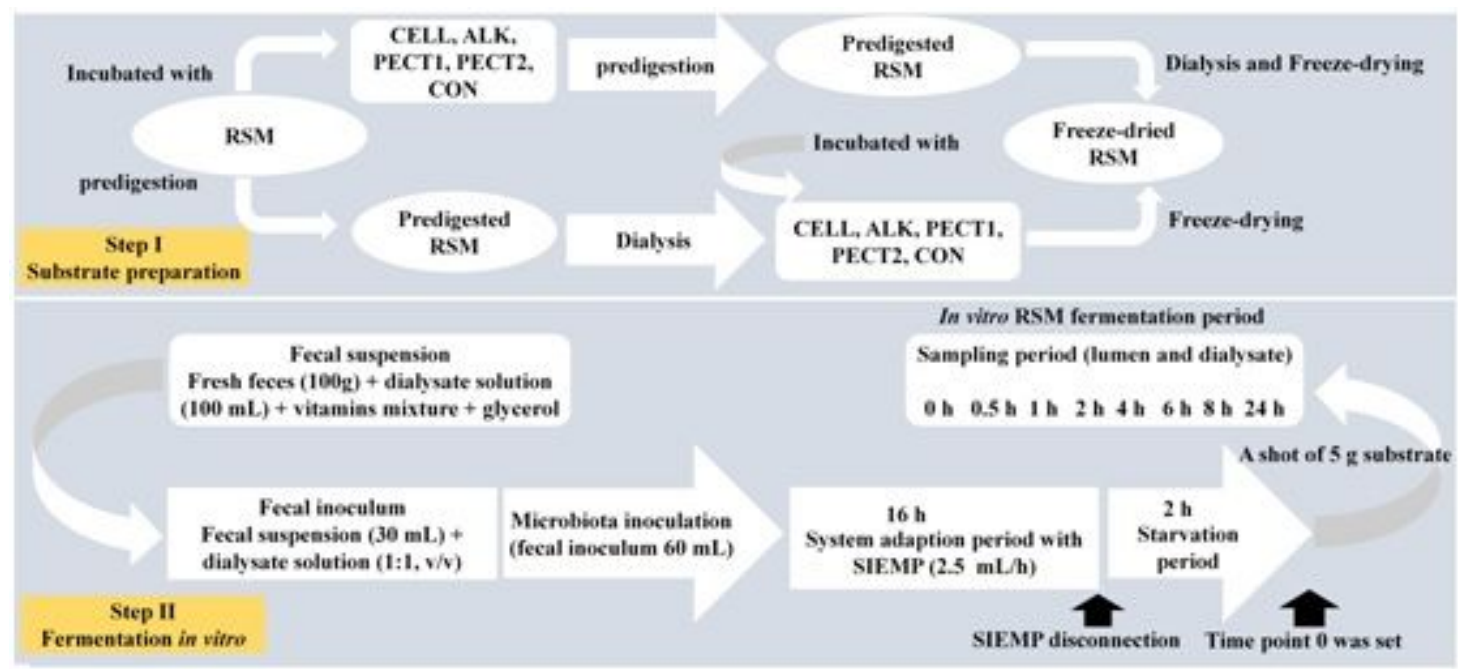

\section{Figure 1}

Schematic experimental setup for fibre fermentation in the Swine Large Intestine in vitro model (SLIM). 


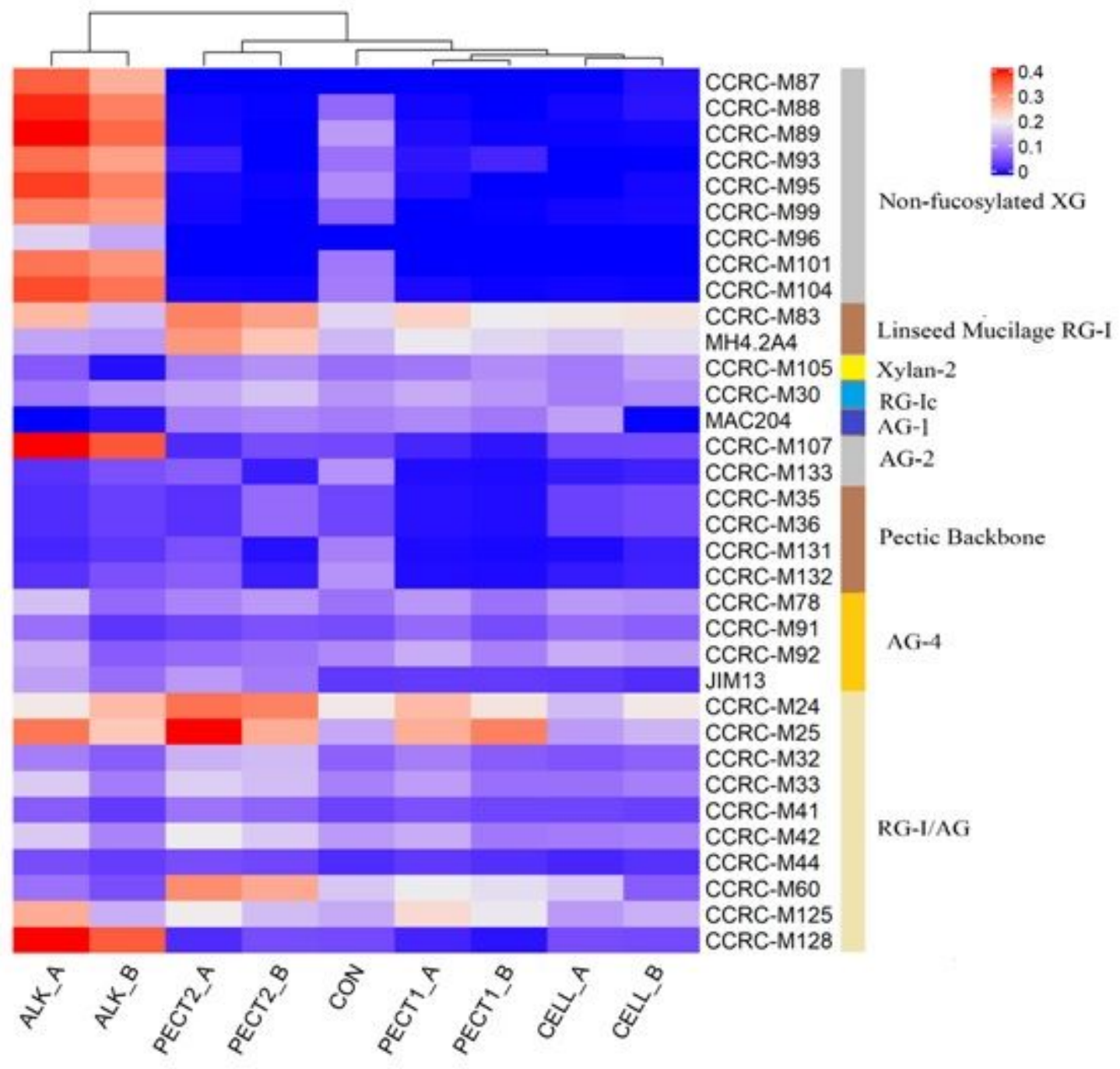

Figure 2

Glycome profiling of non-processed and processed RSM. Thebinding response data of the mAbs are presented as heatmap using a blue-white-red scale indicating the intensityof the ELISA signal (blue, white, and redcolors depict no, medium, and strongbinding, respectively). The mAbs (indicated by their codes) are grouped based on the cell wall glycans they predominantlyrecognize as shown in the panel on righthand side of the figure._A, RSM was treated after predigestion, _B; RSM was treated before predigestion. $X G$, xyloglucan; RG-I, rhamnogalacturonan I; $A G$, arabinogalactan;RG-I/AG, arabinogalactanside chains of RG-I. 


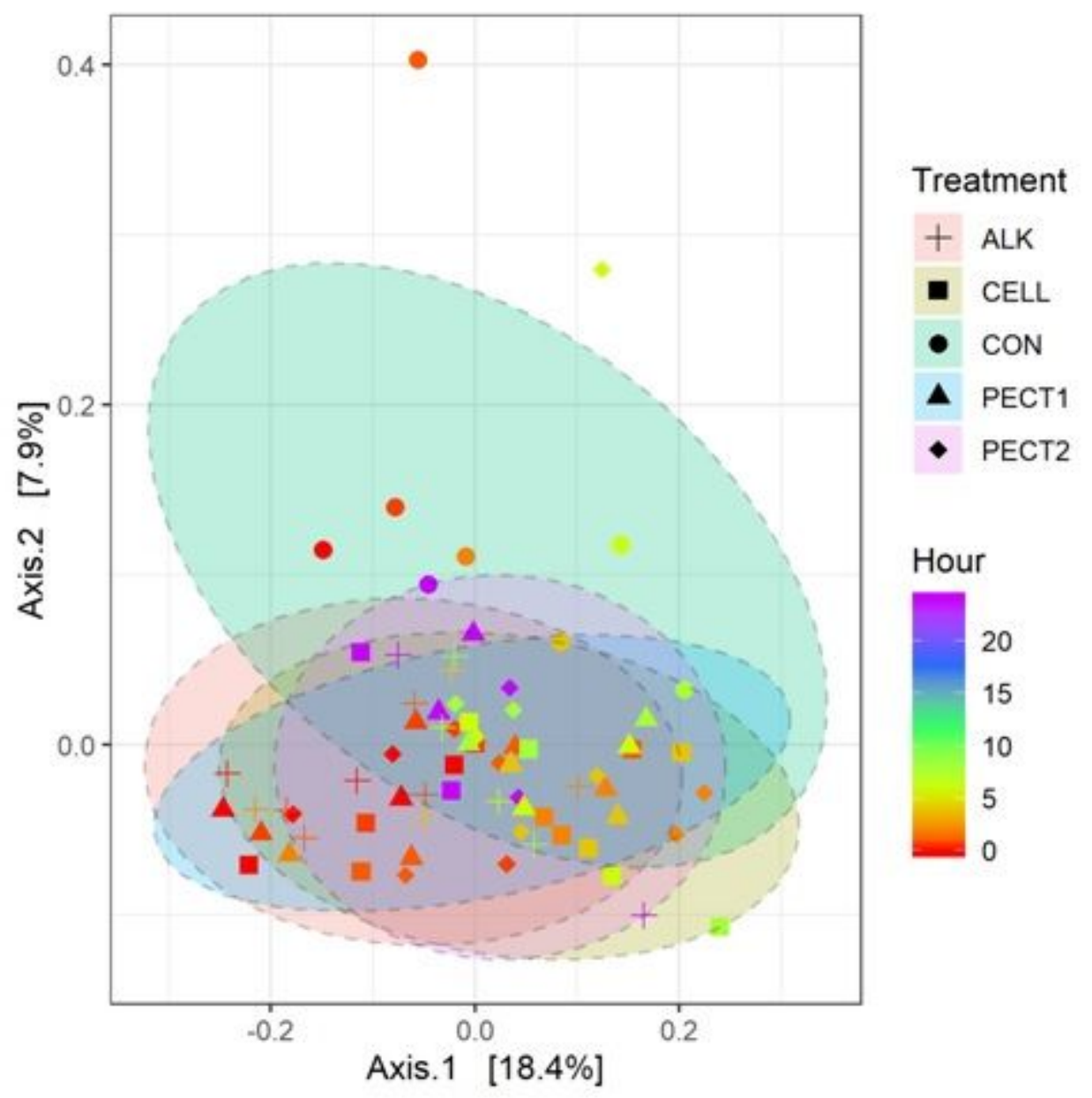

\section{Figure 3}

Principal coordinate analysis (PCOA) plot generated based on the calculated distances in unweighted UniFrac matrix. Samples were grouped by shape and color in terms of treatment and time point they belonged to, respectively: CON(sphere), ALK(plus), PECT1 (triangle), PECT2(diamond),CELL(square); a red-green-purple scale was used to indicate the fermentation time (red and purpledepict start and end of the fermentation period). 

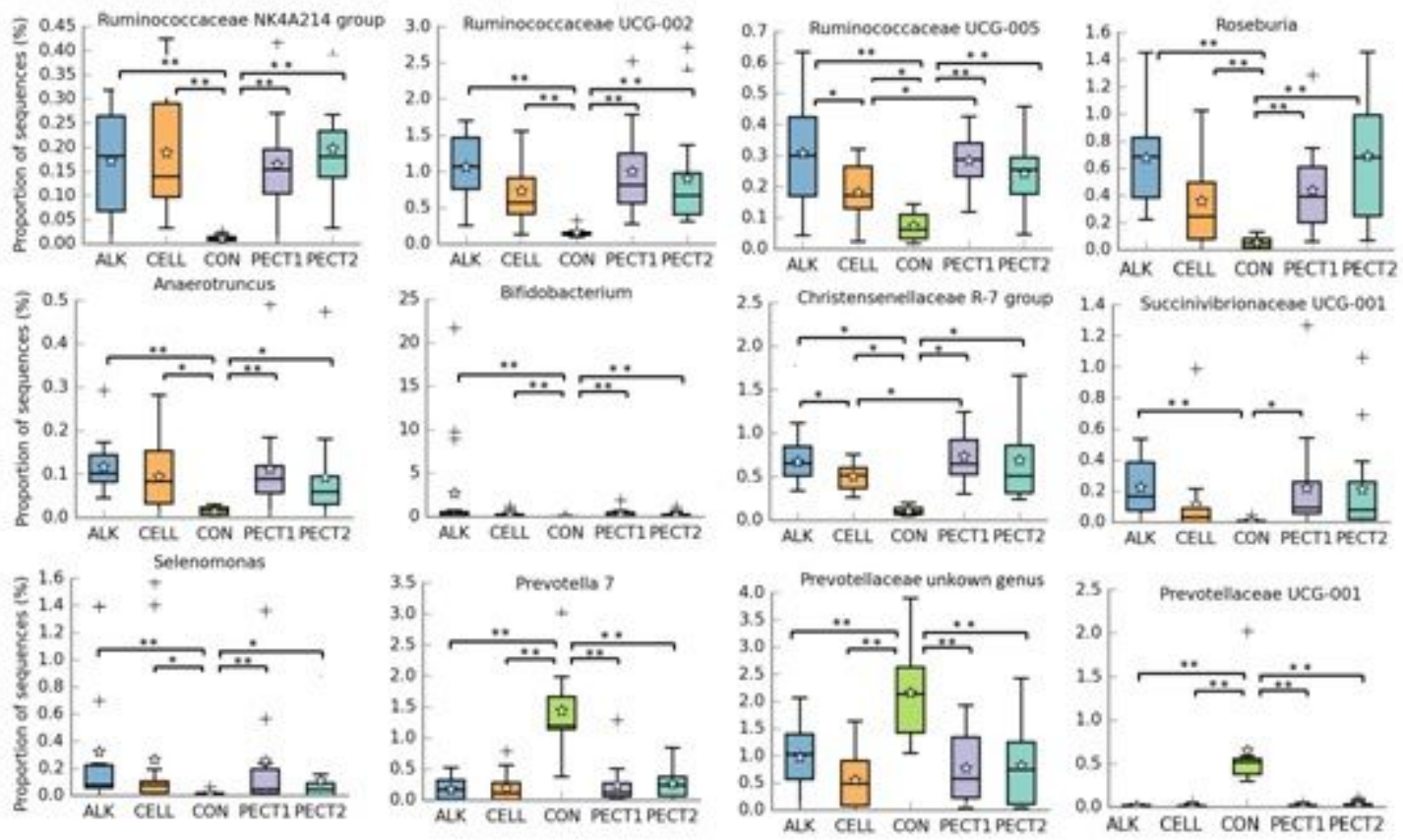

Figure 4

Significantly different relative abundances of microbial generaupon treatment with ALK, CELL, CON, PECT1, and PECT2.* $\mathrm{P}<0.05 ;$ ** $\mathrm{P}<0.01$
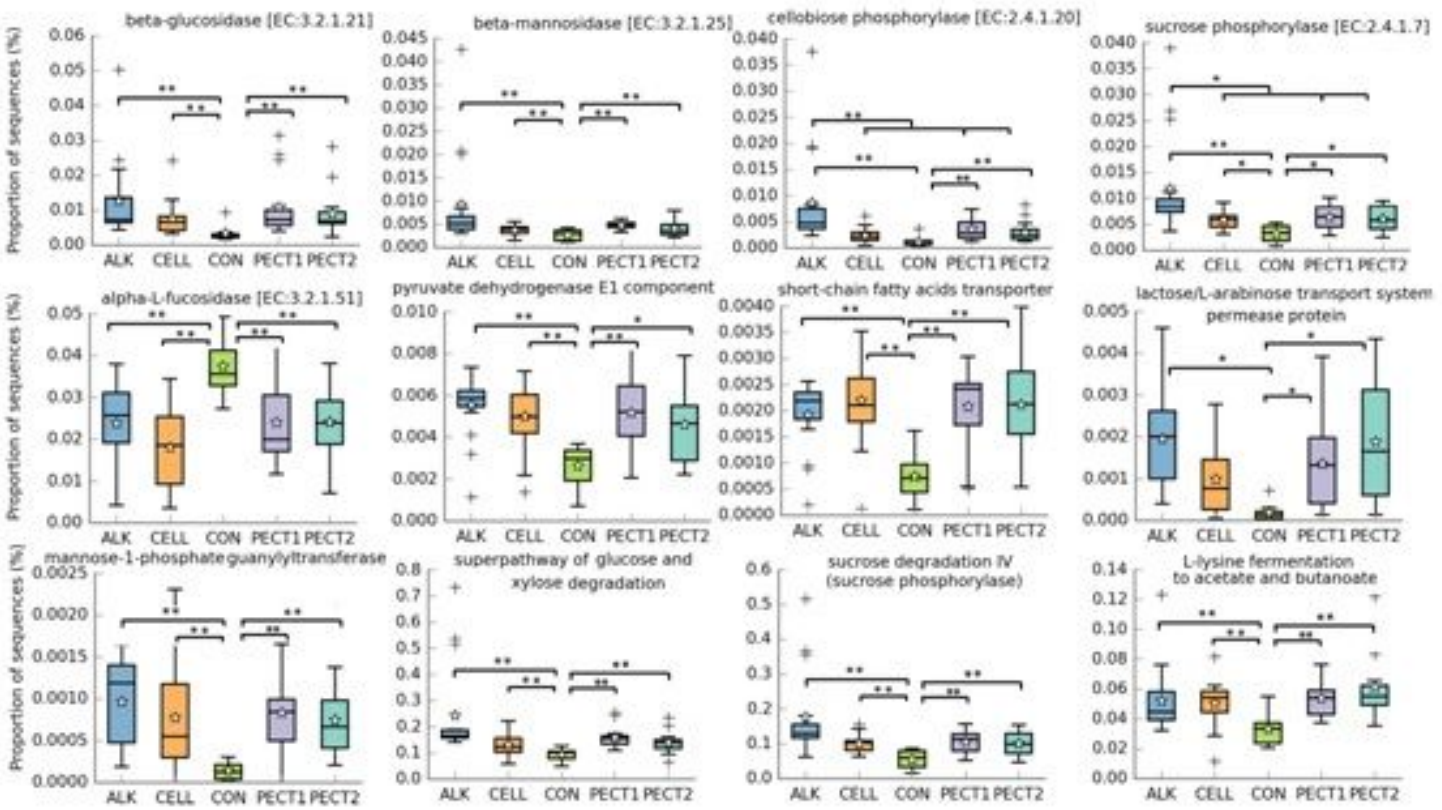

Figure 5

Significantly different metagenomic functions in relative abundance among ALK,CELL, CON, PECT1, and PECT2. * $\mathrm{P}<0.05 ; * \star \mathrm{P}<0.01$. 


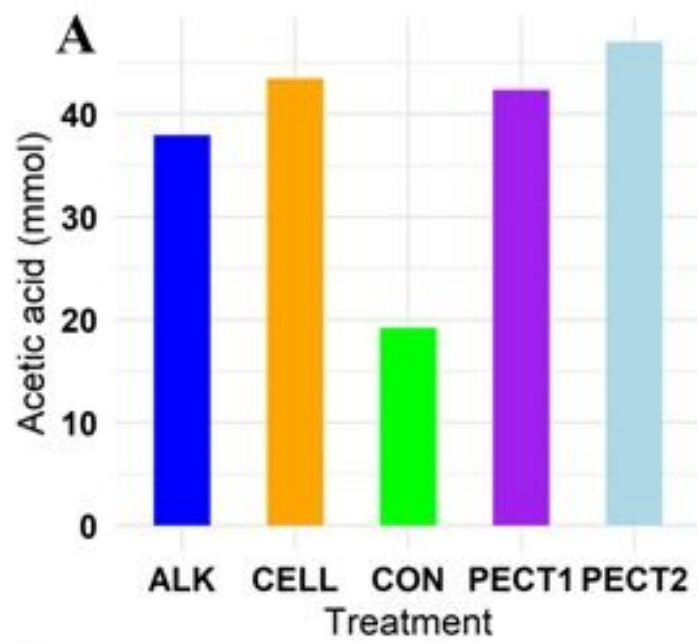

C
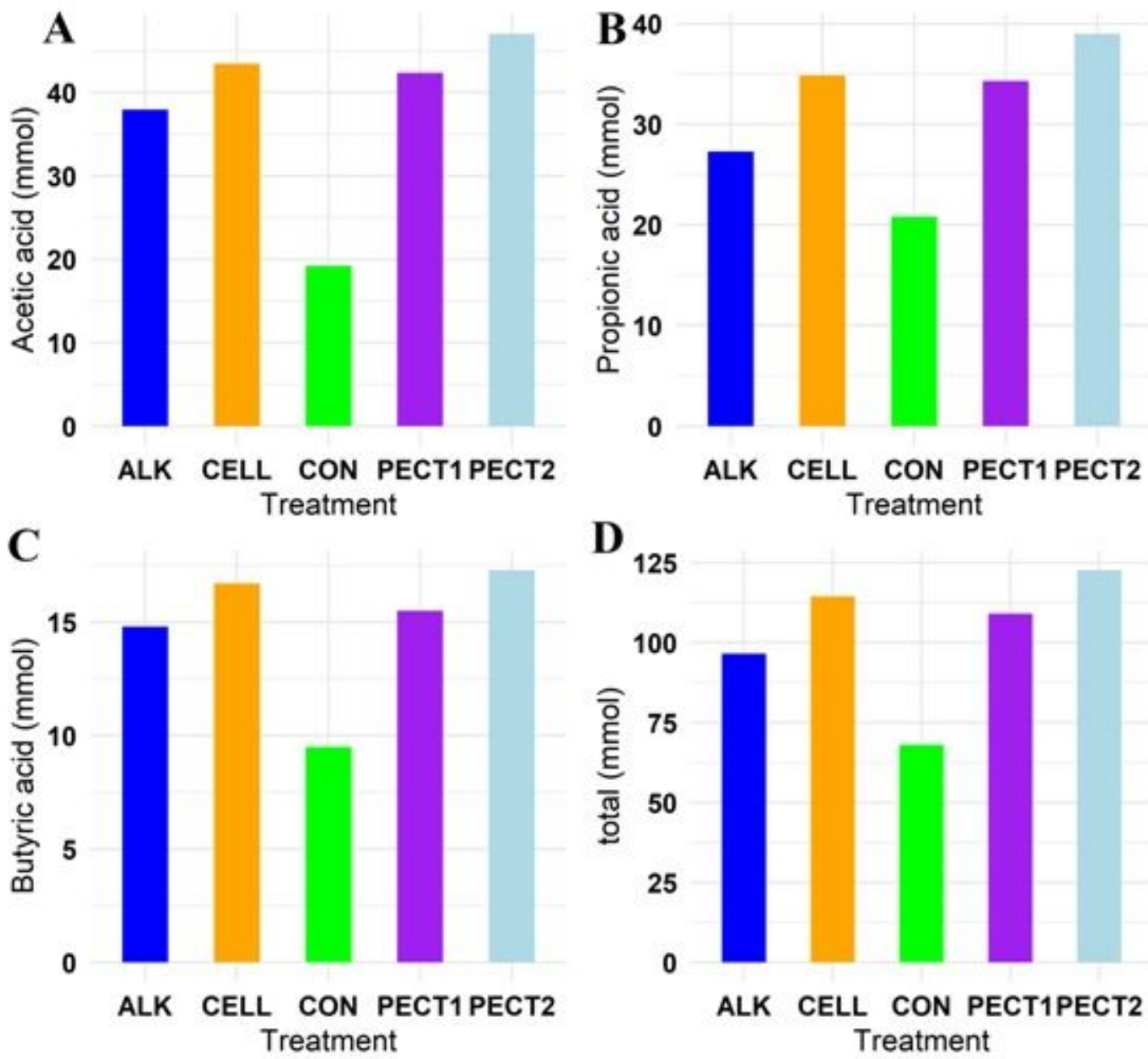

$D_{125}$

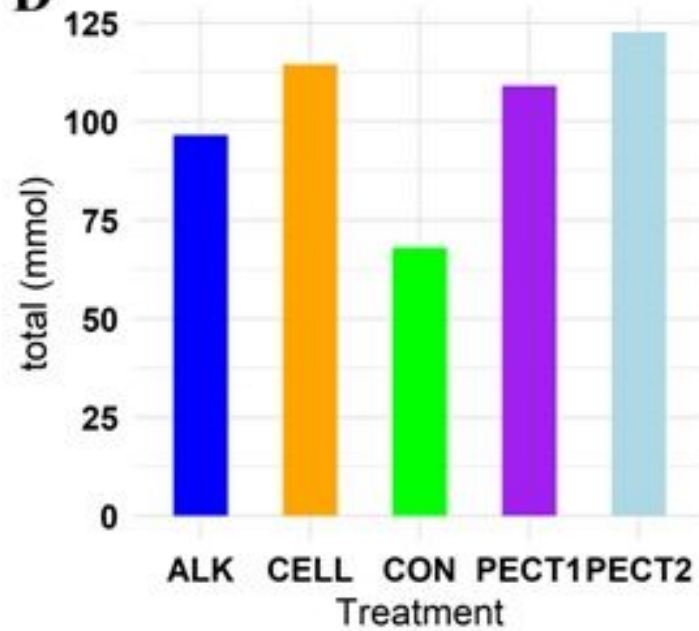

$\mathbf{E}$

\begin{tabular}{|c|c|c|c|c|}
\hline Treatment & Acetic acid & Propionic acid & Butyric acid & total \\
\hline ALK & 2.0 & 1.3 & 1.6 & 1.4 \\
\hline CELL & 2.3 & 1.7 & 1.8 & 1.7 \\
\hline PECT1 & 2.2 & 1.6 & 1.6 & 1.6 \\
\hline PECT2 & 2.5 & 1.9 & 1.8 & 1.8 \\
\hline
\end{tabular}

Figure 6

Cumulative Acetic (A), Propionic (B), and Butyric acid (C), and total short-chain fatty acid (D)production during fermentation of ALK, PECT1, PECT2, and CELL compared to CON. Fold change of short-chain fatty acid in ALK, PECT1, PECT2, and CELL compared to CON (E). 


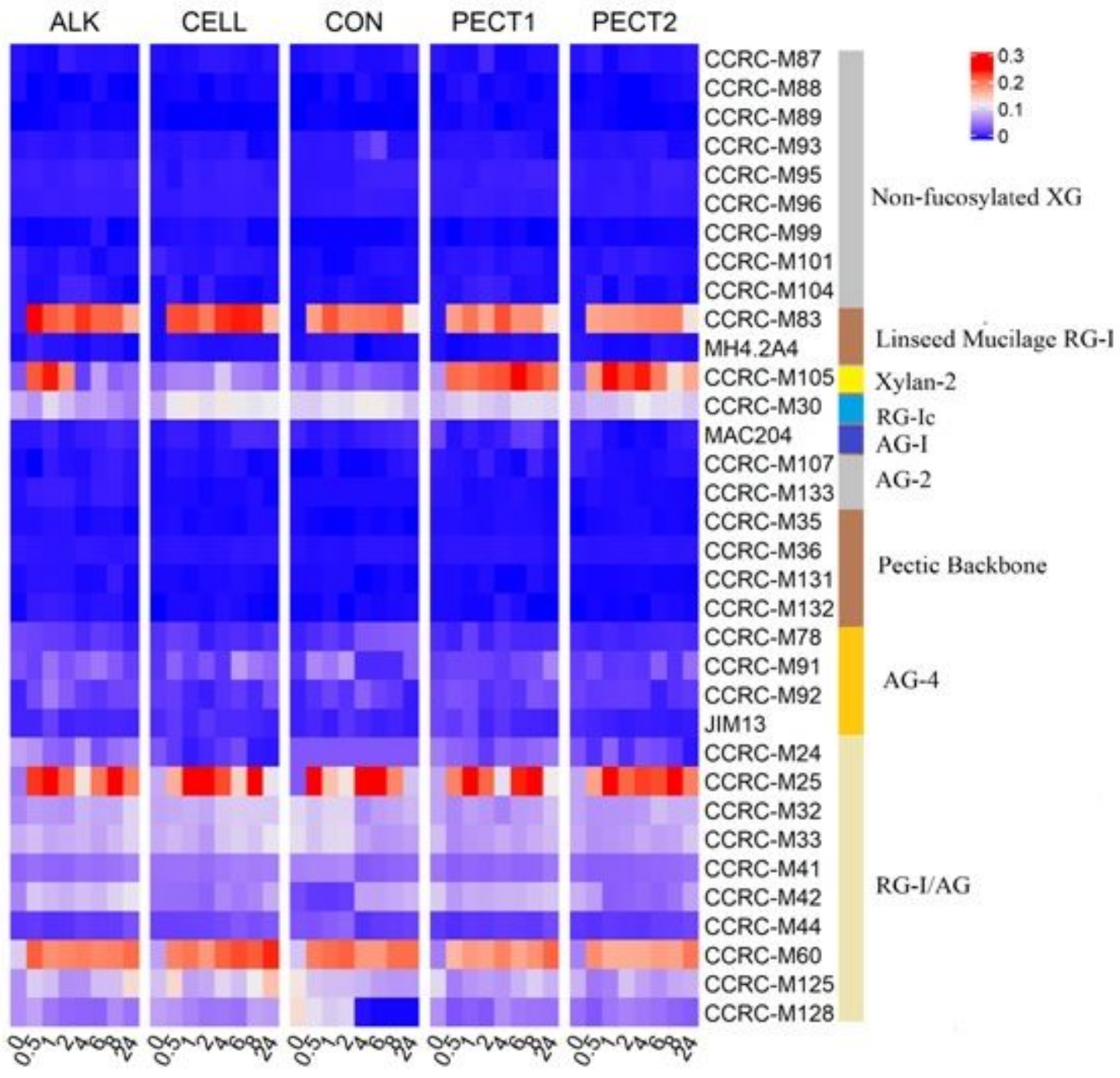

\section{Figure 7}

Glycome profiling of non-processed and processed RSM and lumen digests during in vitrofermentation of ALK, CELL, CON, PECT1, and PECT2 at time point 0, 0.5, 1, 2, 4, 6, 8, and $24 \mathrm{~h}$. Thebinding response data are presented as heatmap using a blue-white-red scale indicating the strength of the ELISA signal (blue, white, and redcolors depict no, medium, and strongbinding, respectively). The mAbs are grouped based on the cell wall glycans they predominantlyrecognize as shown in the panel on the right-hand side of the figure. XG, xyloglucan; RG-I, rhamnogalacturonan I; AG, arabinogalactan; RG-I/AG, arabinogalactanside chains of RG-I. 


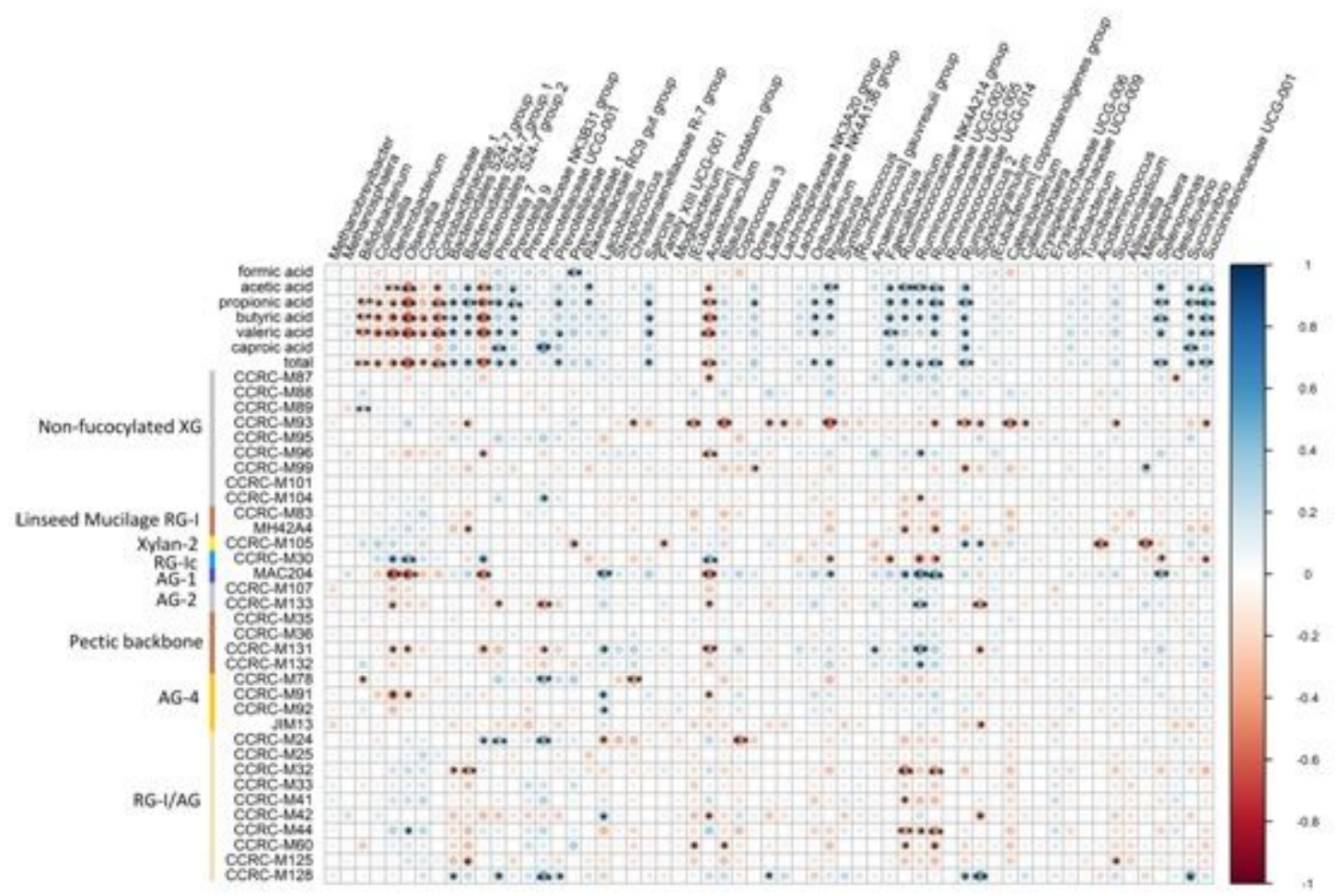

Figure 8

Correlation between core bacterial genera,and SCFAand binding of mAbs. Statistical significance was determined for all pairwise comparisons using Pearson'smethod. $* P<0.05 ; * \star P<0.01$. Size of the circles indicatescorrelation strength, which bigger sizes meaning higher correlations. Blue circles represent positive correlations (correlation coefficients from 0 to 1 ), whereas red circles represent negative correlations (correlation coefficients from 0 to -1).total, total SCFA production. The mAbs are grouped based on the cell wall glycans they predominantlyrecognize as shown in the panel on the left-hand side of the figure. XG, xyloglucan; RG-I, rhamnogalacturonan I; AG, arabinogalactan; RG-I/AG, arabinogalactanside chains of RG-I.

\section{Supplementary Files}

This is a list of supplementary files associated with this preprint. Click to download.

- Suplementalfigures.docx

- Suplementalfigures.docx 